DOI: http://dx.doi.org/10.20435/multi.v21i49.555

\title{
A Unidade de Trauma da Santa Casa de Campo Grande: um estudo de caso no ponto de vista do desenvolvimento local
}

The Santa Casa of Campo Grande Unity of Trauma: a case study in local development viewpoint

Eduardo Fernandes Santos ${ }^{1}$

${ }^{1}$ Graduado em Administração de Empresas. Mestre em Desenvolvimento Local pela Universidade

Católica Dom Bosco (UCDB). Campo Grande, MS. E-mail: eduardofernandessantos@hotmail.com 


\section{RESUMO ABSTRACT}

Este trabalho é fruto de pesquisa realizada Santa Casa de Campo Grande, com o objetivo de demonstrar o papel da Unidade de Trauma no desenvolvimento local. A referida Unidade, ainda em obras, localiza-se em um pavilhão anexo à Santa

Casa para atendimento exclusivo aos usuários do SUS. Os profissionais, colaboradores realizaram estudos para verificar o que será necessário para colocar este complexo em funcionamento e conhecerem o impacto local e regional que esta exercerá. Desse modo, trabalhamos de forma que isso sirva de base para chegarmos a indicadores populacionais no âmbito do desenvolvimento local tendo como prerrogativas a história da Santa Casa, fazendo um breve histórico e mostrando toda a sua estrutura, elencando pontos da saúde e do desenvolvimento local e, então, a Unidade de Trauma em si, todo o estudo que realizamos em relação à estrutura que será desenvolvida, para que essa Unidade entre em funcionamento.
The survey was conducted in the Santa Casa de Campo Grande, in order to demonstrate the role of the Trauma Unit at Local development. The unit is located in an annex pavilion for exclusive service to users of SUS. Professionals conducted studies to see what you need to put this complex operation. In the whole context of this work, we show how the health sector has to develop to do a good job to the population. We work so that it serves as the basis to reach the population indicators in the local development. With the prerogatives the history of the Santa Casa, making a brief history and showing its entire structure, listing health points and local development and then the Trauma Unit itself, the entire study so that this unit is in operation..

\section{KEY WORDS}

reverse logistics

environmental strategy

competitiveness

information system 


\section{INTRODUÇÃo}

A Associação Beneficente de Campo Grande - Santa Casa, na busca de melhoria dos serviços de Saúde Pública, especialmente no atendimento emergencial no Pronto Socorro em vista do número elevado de pacientes com traumas ortopédicos, junto com o Fundo Nacional de Saúde, Ministério da Saúde e a rede municipal de saúde pública, visualizaram a necessidade da evolução da Unidade do Trauma. Reforça essa necessidade o fato de que não está instituída, no município de Campo Grande e no Estado de Mato Grosso do Sul, a rede de atenção ao trauma em ortopedia, além do que existem entraves no fluxo para pacientes que buscam como primeira escolha os serviço da Santa Casa.

A Unidade do Trauma é um projeto em desenvolvimento que prevê desde a subdivisão de serviços especializados que executam processos diferentes até a sua complexidade: fluxos de trabalho, materiais e insumos, estrutura física, visando evitar problemas futuros de funcionamento e de controle de infecção nas unidades e no hospital como um todo, tendo como premissa de análise a RDC n. 50 de 21 de Fevereiro de 2002, da Agência Nacional de Vigilância Sanitária. Para a implantação da Unidade de Trauma, considera-se que o hospital é o único credenciado no Ministério da Saúde como HOSPITAL TIPO III EM URGÊNCIA e está inserido no Plano de Ação da Rede de Atenção às Urgências do Estado de Mato Grosso do Sul.

Em vista do que se propõe, o Administrador Hospitalar deve atuar a partir de uma visão sistêmica, gerenciando planos de ação que contemplem as atividades a serem desenvolvidas e tornando o colaborador mais atuante enquanto parte integrante do processo. Uma vez que os custos dos trabalhos prestados podem influenciar na qualidade e produtividade, devem ser avaliados o tempo, o tipo e natureza do serviço e os recursos utilizados, enquanto procedimentos do planejamento estratégico. 
O planejamento é fixado em objetivos e leva em conta fatores indispensáveis à evolução do hospital sendo então necessário se conhecer as particularidades de cada atividade desenvolvida, visando à eficácia e eficiência do serviço.

0 presente estudo caracteriza-se por uma pesquisa descritiva que visa demonstrar a relevância da implantação da Unidade de Trauma Ortopedia da Associação Beneficente de Campo Grande - Santa Casa, do CNES número 0009717, da ANS número 306428, localizada na rua Eduardo Santos Pereira, 88, Centro, no município de Campo Grande, no Estado de Mato Grosso do Sul, apresentando como objetivo principal: analisar os processos por meio da visão administrativa dos atendimentos que serão realizados na Unidade de Trauma e qual o impacto que o Município de Campo Grande terá com esses atendimentos. Constitui objetivo específico: verificar o perfil dos usuários atendidos na Unidade do Trauma.

Com a abertura da Unidade de Trauma na Santa Casa, o Pronto Socorro da instituição deverá ter uma diminuição no atendimento tendo em vista que os usuários que tiverem o diagnóstico de trauma serão encaminhados para essa nova unidade. Isso implica melhoria no atendimento das outras especialidades que derem entrada no hospital, e com isso, os profissionais deverão realizar o atendimento com mais humanidade e agilidade no processo.

Por fim, será realizado estimativa de dimensionamento de recursos humanos, embasado na legislação pertinente a cada profissão/área e considerada essencialmente a proposta assistencial de cada Serviço. Assim, o administrador hospitalar poderá exercer suas atribuições de forma especializada, representando as necessidades para com o hospital, tendo então como função criar, em sua administração, métodos participativos que melhoram significativamente a democratização das tomadas de decisões, estabelecem melhor satisfação no trabalho e aumento da produtividade. 
0 referido estudo segue realizando uma contextualização do seu objeto, explanando um pouco da Santa Casa e sua história desde o momento de sua fundação até os dias de hoje. Indicam-se os serviços que ela presta à sociedade, junto com seus indicadores de produção hospitalar, para que se possa ver a grandiosidade dos trabalhos prestados para a população.

Percorreu-se o caminho da administração hospitalar abrangendo os hospitais no Brasil, privilegiando o que é um hospital filantrópico, como é o caso da Santa Casa de Campo Grande.

Este estudo mostra também a classificação hospitalar no Brasil e como ela é realizada. A Santa Casa realiza a mesma classificação que muitos outros hospitais do Brasil, levando em conta os fatores que fazem com que a população tenha atendimento humanizado e de qualidade.

À medida que a pesquisa foi sendo desenvolvida, salientou-se o aspecto econômico das questões relacionadas à saúde, tendo em vista que a Unidade de Trauma ortopédico está sendo analisada, tanto em seu lado organizacional quanto econômico, indicando os seus processos internos que deverão ser desenvolvidos para o seu funcionamento.

O desenvolvimento local está diretamente relacionado com a área da saúde. Devido a isso, indica-se a maneira como tal relação ocorre, como se dá a produção da saúde, como ela é desenvolvida, administrada para, desse modo, verificar o crescimento institucional da Santa Casa, considerando os fatores externos demandados pela população. Dentre os fatores, consta como é realizada a gestão do Sistema Único de Saúde (SUS), tendo em vista que a Unidade de Trauma ortopédico atenderá $100 \%$ o SUS.

A consideração da demanda advinda da população é fator importante para o desenvolvimento local e, por isso, foram indicadas várias formas em que esse modo de conceber o desenvolvimento se faz presente no dia a dia. Em vista disso, o trabalho de pesquisa em si pode ser considerado um estudo de caso em 
desenvolvimento local. Para efeito de compreensão da complexidade que é a Santa Casa, foram detalhadas que atividades desenvolvidas serão desenvolvidas na Unidade de Trauma ortopédico. Igualmente foram descritos os fluxos necessários de cada atividade, visando ao pleno atendimento à população.

Dentre os estudos acima citados, fez-se uma previsão dos serviços de lavanderia e cozinha, pois eles serão realizados pela estrutura já existente na instituição.

Por fim, realizou-se a análise dos dados coletados e uma estimativa de custo em percentual da unidade em estudo, para que se pudesse verificar em quais áreas a Santa Casa de Campo Grande terá que realizar seus trabalhos com eficiência e qualidade.

\section{SANTA CASA E SUA HISTÓRIA}

Com a aprovação em estatuto em 3 de junho de 1919, institui-se a Associação Beneficente de Campo Grande, entidade mantenedora da Santa Casa, a fim de suprir a deficiência de atendimento na cidade, que não dispunha de qualquer tipo de assistência médico-hospitalar (SANTA CASA, s/d).

Inicialmente foi construído, em uma área de $61.725,00 \mathrm{~m}^{2}$, um pequeno pavilhão com 40 leitos - esse pequeno hospital passou a assumir toda a responsabilidade do atendimento à saúde na cidade e regiões circunvizinhas. Devido ao seu desempenho e resolutividade, foi gradativamente ampliando o número de atendimento e gerando, consequentemente, a necessidade de aumento de sua estrutura física (SANTA CASA, s/d).

Atualmente o espaço físico é de $42.665,87 \mathrm{~m}^{2}$, com uma capacidade instalada de 685 leitos ativos e está em construção um novo Anexo Hospitalar de mais $6.124 \mathrm{~m}^{2}$ que abrigará a nova Unidade de Traumatologia que contará com mais 110 leitos novos leitos para atendimento de uma demanda crescente de pacientes da ortopedia (SANTA CASA, s/d). 
A Santa Casa de Campo Grande constituiu-se por sua estrutura física e quantidade de serviços prestados na unidade Hospitalar do Estado de Mato Grosso do Sul, sua relevância é reconhecida pela comunidade e poder público, que habilitou inúmeros serviços especializados para a capital, 78 municípios do Estado e até mesmo outros Estados e países vizinhos (SANTA CASA, s/d)

A demanda da Santa Casa de Campo Grande está diretamente ligada aos serviços acima relacionados. Em vista disso, os leitos de internação são divididos em internação cirúrgica, clínica que são disponibilizados para pacientes que necessitam do centro cirúrgico e pós-cirúrgico.

Os leitos de obstetrícia cirúrgica e clínica são divididos em pacientes que necessitem da realização de partos e pós-partos.

Nos casos de leitos de internação em pediatria cirúrgica e clínica, estes são disponibilizados para pacientes crianças que necessitem de atendimento cirúrgico e pós-cirúrgico.

Os leitos de unidade de isolamento são disponibilizados para pacientes que obtêm um diagnóstico que possa, de alguma forma, transmitir algum tipo de vírus ou bactérias que para outros pacientes.

Os leitos de UTI adulto, neonatal e pediátrico são disponibilizados para pacientes que necessitam de atendimento de unidade de terapia intensiva. Nos casos de leitos de unidade intermediária neonatal, estes são disponibilizados para pacientes bebês recém-nascidos que necessitem de atendimento hospitalar.

Os leitos para o atendimento de pacientes crônicos são disponibilizados para pacientes que tenham algum diagnóstico de uma doença crônica, para se restabelecerem.

Nos casos dos leitos para pacientes da psiquiatria, estes são disponibilizados para aqueles com diagnóstico psiquiátrico que necessitem de cuidados especiais para o restabelecimento. 


\section{ADMINISTRAÇÃO HOSPITALAR - EMPRESA SEM FINS LUCRATIVOS}

Entidades sem fins lucrativos são firmas organizadas para fornecer bens filantrópicos ou serviços comunitários e que obtêm suas receitas, em grande parte, por doações. Embora muitas se enquadrem nessa categoria, tal definição não é conceitualmente adequada nem faz uma distinção econômica importante. Por um lado, muitas empresas sem fins lucrativos atendem aos mais abastados, e muitas prestam atendimento competindo com organizações com fins lucrativos. Muitas obtêm a maior parte de suas receitas da venda de bens e serviços a preços similares aos de suas concorrentes que têm fins lucrativos. As empresas sem fins na assistência à saúde obtêm mais de $90 \%$ de suas receitas com "vendas e recebimentos" (FOLLAND; GOODMAN; STANO, 2008).

\subsection{Origem da palavra hospital}

Segundo Cherubin e Santos (2002) a literatura hospitalar mostra que há divergência entre os especialistas sobre a origem da palavra hospital. Para alguns, deriva da palavra latina hospedes que significa hóspede. Nesse caso, o hospital seria uma instituição que acolhe alguém.

A segunda definição refere-se ao termo hospitium, que significa o local onde se mantém alguém hospedado. Hospitais é um qualificador de hospitium e significa hospitaleiro. No dicionário Aurélio, está escrito que hospital vem de hostitale que, em latim, significa hospedaria. Atualmente a palavra hospital decorre da própria definição de saúde que tem por finalidade "perfeito bem-estar físico, social e mental, e não a simples ausência de doenças", tendo como função atender a população em seus domínios (CHERUBIN; SANTOS, 2002). 0 atendimento da população é um dos fatores relevantes para a humanização hospitalar, tendo em 
vista que todas as instituições de saúde estão absorvendo essa metodologia, pois ela traz uma melhoria para os pacientes, diminuindo assim o retorno deles ao hospital.

\subsection{Hospitais filantrópicos}

O setor hospitalar filantrópico no Brasil é responsável por cerca de um terço dos leitos existentes no País, constituindo-se em importante prestador de serviços para o Sistema Único de Saúde (SUS) e para o setor da saúde suplementar. É objeto de programas especiais do Ministério da Saúde, das áreas de governo de arrecadação de tributos e contribuições e de instituições da área econômica, tais como o Banco Nacional de Desenvolvimento Econômico e Social (BNDES). Nesse sentido, esse setor requer ser mais bem conhecido para formulação de políticas governamentais visando ao seu desenvolvimento e maior integração ao sistema de saúde brasileiro, nas esferas pública e privada (PORTELA et al., 2004).

Pela regulamentação vigente, são consideradas filantrópicas as entidades portadoras do "Certificado de Entidade Beneficente de Assistência Social", concedido pelo Conselho Nacional de Assistência Social (CNAS), órgão colegiado subordinado ao Ministério do Desenvolvimento Social e Combate à Fome. Várias condições são exigidas para a obtenção desse Certificado. Para os hospitais, destacam-se duas alternativas previstas pelo Decreto no $4.327 / 2002: 3$, a oferta e efetiva prestação de $60 \%$ ou mais de internações são SUS; e, no caso de o gestor local do SUS declarar impossibilidade de contratação de $60 \%$ de internações, a entidade aplicar um percentual da receita bruta em gratuidade variando entre $20 \%$ e $5 \%$, na dependência do efetivo percentual de atendimento ao SUS. 0 referido decreto prevê uma terceira alternativa para a obtenção do Certificado, a classificação do hospital como estratégico para o SUS. Entretanto a definição de critérios para tal classificação e de uma lista de hospitais neles enquadrados, 
estabelecida pelo Ministério da Saúde em 2002, foi revogada no início de 2003 (PORTELA et al., 2004).

\subsection{Gestão hospitalar}

Para que se consiga realizar um atendimento com excelência, é preciso entender que os pacientes não finalizam o atendimento após obter alta hospitalar, e, sim, com o tratamento pós-hospitalar, no que se refere aos serviços externos da instituição, para verificar se, após a alta, o paciente obteve alguns outros sintomas.

Segundo a OMS, o hospital é parte integrante de um sistema coordenado de saúde cuja função é atender a comunidade, disponibilizar a assistência à saúde tanto curativa quanto preventiva, incluindo serviços extensivos (assistência à família a domicílio) e ainda um campo de formação e pesquisa para os profissionais da área da saúde (CHERUBIN; SANTOS, 2002).

Em muitos casos, o atendimento aos pacientes externos são realizados por voluntários que prestam serviços em instituições filantrópicas que tenham qualificação técnica para tal.

\subsection{Planejamento e análise de custos}

A Santa Casa (2014) realiza periodicamente a análise das etapas previstas no planejamento estratégico no sentido de avaliar cada serviço executado e quais deverão ser realizados segundo o plano de ação, visando primordialmente à qualidade dos trabalhos e à diminuição nos gastos.

A relação entre planejamento hospitalar e gerenciamento de custos representa um dilema relacionado à falta de recursos e ao excesso de demanda. Dessa forma, o administrador deve atuar a partir de uma visão sistêmica gerenciando planos de ação que contemplem os projetos e atividades a serem desenvolvidos. Os projetos não correspondem às ações rotineiras, e sim na criação de 
critérios de avaliação: de orçamento, de responsabilidade e tempo na execução de cada atividade (TASCA, 1999 apud BRASIL, 2000).

\subsection{0 administrador hospitalar reestruturando}

Para que ocorra uma organização nos procedimentos que serão desenvolvidos na nova unidade, a administração do hospital estará se reestruturando organizacionalmente para que não ocorra prejuízo no atendimento dos pacientes do SUS.

A reestruturação do hospital ou de um setor hospitalar requer um desempenho que se fundamenta em três razões: as fases econômicas das empresas; estratégia de capacitação e remuneração disponibilizando incentivos financeiros; a influência de fatores políticos. (CAMARGO, 2000 apud BRASIL, 2000).

\section{A SAÚDE E O DESENVOLVIMENTO LOCAL}

O mercado da saúde no Município de Campo Grande está em um processo de estruturação e desenvolvimento de habilidades e processos que desenvolvam a população junto com agentes de saúde. Esse processo está sendo desenvolvido de forma integrada com a população, por intermédio de políticas públicas visando à publicidade e à disseminação no âmbito de contribuir para que ocorra um auxílio no combate a doenças e a outros fatores que sejam preponderantes à saúde da população.

\subsection{Produção de saúde}

Cada dia mais, os hospitais estão realizando estudos para o aprimoramento de seus processos no intuito de alinhar e controlar os seus processos internos para que a realidade econômica das instituições realize com êxito as suas atividades e a sua produção seja efetiva e com regulação dos processos. 
A produção de saúde é uma preocupação central para os economistas da saúde e para as políticas públicas. Considere que o papel da assistência à saúde na sociedade, inclusive o atendimento prestado pelos médicos, é, em última análise, uma questão de produção. Qual é a contribuição da assistência à saúde para o estado de saúde da população? Podemos então perguntar qual é a melhor maneira de se produzir e distribuir a assistência à saúde? (FOLLAND; GOODMAN; STANO, 2008).

Uma função produção descreve a relação entre insumos e produtos. 0 estudo da função produção de saúde requer que nos perguntemos sobre a relação entre os insumos da assistência à saúde e a saúde. As respostas que os economistas e historiadores da medicina oferecem a essa pergunta surpreendem a muita gente. Primeiro a contribuição da prestação dos serviços de saúde para o progressivo declínio das taxas de mortalidade das populações foi provavelmente negligenciada pelo menos até uma boa parte do século XX. Segundo, embora a contribuição marginal, em certos casos pode ser pequena (FOLLAND; GOODMAN; STANO, 2008).

\subsection{0 papel histórico da medicina e da assistência à saúde}

Muitos historiadores da medicina concordam que as intervenções médicas prestadas desempenharam apenas um pequeno, talvez desprezível, papel no declínio histórico da taxa de mortalidade das populações. A medicina eficaz é um tanto recente, e a prestação de intervenções médicas eficazes numa escala suficiente para afetar os indicadores de saúde da população provavelmente só apareceram no século XX. Embora a magnitude de outras causas do declínio da mortalidade ainda seja objeto de discussão, é óbvio que um papel maior, um dos mais significantes, pode ser atribuído às medidas de saúde pública e à difusão do conhecimento das causas de doenças. No entanto vários pesquisadores dessa área atribuem a maior parcela de crédito às melhorias ambientais, 
particularmente ao aumento da oferta de produtos alimentícios decorrente das revoluções agrícola e industrial (FOLLAND; GOODMAN; STANO, 2008).

\subsection{Desenvolvimento Local}

O desenvolvimento local é fator importante para a Unidade de Trauma ortopédico, e por isso o estudo mostra o que é preciso desenvolver e investir para que a referida unidade entre em funcionamento, disponibilizando leitos de internação e vários outros serviços para atender a enorme demanda advinda da população. Nesse sentido, a Santa Casa (2014) vem desenvolvendo estudos populacionais que permeiam os trabalhos da instituição, para verificar o impacto econômico e social que a Unidade de Trauma ortopédico irá trazer para Campo Grande e o Estado de Mato Grosso do Sul.

0 termo desenvolvimento local se encontra em evidência nos mais variados segmentos, seja tecnológico, urbano, social, seja internacional. Percebe-se que a palavra desenvolvimento vem sofrendo transformações e agregando conceitos como o desenvolvimento regional, subdesenvolvido, desenvolvimento sustentável, Desenvolvimento Local, dentre outros.

Após a revolução industrial, o desenvolvimento passou a ser visto, comumente, como sinônimo de progresso, capaz de gerar riqueza às cidades, oportunizar novas formas de trabalho e desenvolvimento, tanto para o indivíduo quanto para a localidade. Contudo vincular o desenvolvimento de um determinado local, simplesmente com aglomeração de fábricas e indústrias nele encontradas, é um equívoco, que, na atual conjuntura, não se pode cometer.

0 desenvolvimento já foi associado somente à economia, contudo, como salienta Souza (1997, p. 06), “[...] o desenvolvimento não deve ser entendido como sinônimo de desenvolvi- 
mento econômico". No contexto globalizado em que a sociedade tem vivido, permeado por influências externas, status e muitas vezes desvalorização de determinados locais, faz-se necessário um referencial teórico que subdivide estudos que façam emergir o posto dessa realidade.

A análise do presente estudo utilizou-se das teorizações acerca do conceito de Desenvolvimento Local, que parte do ser humano, como ponto central, inserido socialmente em um local com suas dificuldades e potencialidades (ÁVILA, 2000).

Considerando que, na perspectiva de Desenvolvimento Local, devem-se ponderar as características do local e ter um olhar voltado não apenas pela lente econômica, é preciso se atentar para o atendimento das necessidades locais no sentido humano, ou seja, a riqueza da solidariedade, o pertencimento, protagonismo comunitário, dentre outras (ELIZALDE, 2000).

Pensar em Desenvolvimento Local é pensar no local como um campo de intervenção particular. Projetar ou traçar uma meta de desenvolvimento a partir de outros lugares, de outras experiências e levar para ser executado no local foge totalmente da perspectiva dessa abordagem (ÁVILA, 2000).

Estudar o local pela perspectiva do Desenvolvimento Local é considerado como singular. Observar suas peculiaridades é fundamental no Desenvolvimento Local, deve-se analisar uma comunidade com diversas potencialidades e diferentes protagonistas e a dinâmica social local. Buscar compreender como a cultura é expressa e incorporada nos hábitos e na rotina dos indivíduos em um local específico também é inerente quando se analisa determinado local pela perspectiva do Desenvolvimento Local, pois segundo Kliksberg, (2002, p.122-3): “[...] a cultura incide claramente sobre o estilo de vida dos grupos sociais".

Dentre os movimentos que influenciaram e contribuíram para a teorização do Desenvolvimento Local podem ser citadas as cartas elaboradas pela Organização das Nações Unidas no pós- 
-guerra (1945) e os movimentos ambientais, como a Conferência das Nações Unidas realizada em Estocolmo em 1972. Tais Movimentos projetaram "[...] um novo olhar sobre a dinâmica dos fluxos econômicos, que repousa sobre a possibilidade de promover autodeterminação das populações locais [...] atores sociais [...] ruptura dos círculos viciosos do mau desenvolvimento" (FONTAN, VIEIRA, 2011, p. 39).

No Desenvolvimento Local, não se considera mais a visão de níveis verticais, mas horizontais, em que o estado e a comunidade interagem em níveis de conhecimentos de mesmo valor, cuja ênfase é dada às pequenas localidades, consideradas a partir de seus valores endógenos, articulados com os fatores exógenos, suas tradições e identidades.

Para se pensar em Desenvolvimento Local, devem-se considerar as potencialidades de cada local, tais como solidariedade local, cultura, patrimônio material e imaterial, e entender que esse local deve estar articulado com outras regiões. É preciso ponderar as potencialidades locais e reconhecer que a sociedade está cada vez mais complexa e interligada, exigindo, assim, articulações permanentes junto a diferentes organizações (LE BOURLEGAT, 2011).

O Desenvolvimento Local não propugna o isolamento. Ao contrário, o agente de desenvolvimento deve atentar-se aos perigos do localismo, em que se supervaloriza uma localidade que se considerada autossuficiente, sem se relacionar e articular com outros locais.

\subsection{Território e territorialidade}

Ao longo da história da humanidade, é possível perceber que as pessoas buscaram e ainda buscam viver em comunidade, tribos, grupos etc. por diversas razões, seja por motivo de sobrevivência, interesses comuns, sentimento de pertença, seja por necessidade passageira. 
Para o entendimento do Desenvolvimento Local, é inerente conhecer as características físicas, culturais, potencialidades, entraves, atores sociais, suas redes, porque cada local é naturalmente peculiar. Isso reforça a perspectiva de que as comunidades, quando se encontram emponderadas, possuem inúmeras possibilidades para a gestão local, utilizando de suas experiências, iniciativas e articulação com diversas instâncias para alcançar seus objetivos. Nesse sentido, as comunidades buscam a '"metabolização' comunitária de insumos e investimentos públicos e privados externos, visando à busca de soluções para os problemas, necessidades e aspirações de toda ordem e natureza que mais direta e cotidianamente lhe dizem respeito" (ÁVILA, 2000, p. 68).

Segundo Albagli e Maciel (2004), há de se ter a percepção da existência de uma conexão entre as extensões socioculturais e territoriais, tanto em escala individual quanto em grupos sociais, esta desponta em diversas escalas geográficas. Importante também a contribuição de Santos (1994) com as questões que envolvem a territorialidade ao apontar as mudanças que ocorrem nos territórios com o passar dos anos, englobando as rápidas mudanças arquitetônicas nos locais, a exemplo de novas construções, que hoje são realizadas em escalas e modificam um local que representava características daquele local.

É de extrema importância compreender o quanto estão imbricadas as relações entre as pessoas e os territórios nos quais elas estão inseridas, pois a dinâmica social e as representações sociais e de poder dizem muito a respeito de um local. Na mesma perspectiva, Santos (2002) chama a atenção para a análise histórica do território, pois este foi tecido por vários atores, um território "híbrido" que, mesmo em transformação, possui sua história, e esta não pode ser negligenciada. Dele há de se realizar uma "análise social", ou seja, buscar conhecer a dinâmica de determinado local, pois ele não surgiu sem uma história, há de se pensar como o território foi formado, como foram tecidas as 
relações nele estabelecidas, os objetivos das pessoas que moravam naquele território, a cultura, os hábitos o cotidiano de quem vivia no local.

No olhar de Raffestin (1993, p. 160), entende-se como territorialidade um "conjunto de relações que se originam em um sistema tridimensional, sociedade, espaço e tempo em vias de atingir a maior autonomia possível e compatível com os recursos do sistema", ou seja, a territorialidade ocorre quando os indivíduos não somente se apoderam do território, mas dele fazem parte.

\subsection{Espaço, local e lugar}

É muito comum em um contexto informal a utilização das palavras; espaço, local e lugar serem utilizadas como sinônimos, porém, elas não têm o mesmo significado. Ao que concerne ao Desenvolvimento Local, é fundamental compreender as diferenças desses vocábulos, considerando que têm diferentes funções e interpretações (ÁVILA, 2000).

O espaço se apresenta como um ambiente construído dialeticamente. Os significados e representações surgem a partir das ações e sentidos abstratos dados pela comunidade que o habita. $\mathrm{Na}$ mesma perspectiva, Martins (2002) aponta para "prática espacial" que ocorre no espaço por meio das "representações sociais".

Ao visualizar o sentido de local no que se refere ao Desenvolvimento Local, Martins (2002, p. 54) destaca que tal acepção relaciona-se ao intuito de acompanhar melhor determinado local, valorizar "a escala das inter-relações pessoais da vida cotidiana, que sobre uma base territorial constroem sua identidade".

Seguindo o trajeto do espaço para local, chega-se às concepções que envolvem o lugar. No olhar de Tuan (1980, p. 387), "o lugar encarna as experiências e aspirações das pessoas. 0 lugar não é só um fato a ser explicado na ampla estrutura do espaço, ele é a realidade a ser esclarecida e compreendida sob a perspectiva 
das pessoas que lhe dão significado". Entende-se que o lugar foi vivenciado pelo indivíduo em um contexto concreto, e este ampliou tais relações para o campo simbólico espacial.

É no lugar que as pessoas passam a fazer referência a determinado território, por se remeter àquele com seus simbolismos e características criadas a partir do físico para o subjetivo. Assim, deve ser valorizada a identidade local, cultura e aspectos que fazem de um local um lugar. Desse modo, o lugar cativa indivíduos, que por ele passam, fomentando o pertencimento, relações de solidariedade possíveis articulações em escalas mais amplas.

\section{UNIDADE DE TRAUMA ORTOPEDIA}

A Associação Beneficente de Campo Grande - Santa Casa, na busca dos serviços de Saúde Pública, especialmente no atendimento emergencial em Pronto Socorro, em vista do número elevado de pacientes com trauma ortopédico, junto com instituições de saúde pública, visualizou a necessidade da evolução da Unidade do Trauma. Salienta-se que não está constituída no Município de Campo Grande e no Estado de Mato Grosso do Sul a rede de atenção ao Trauma ortopédico. Existem entraves organizados de fluxo para pacientes que buscam como primeira escolha o serviço da Santa Casa.

A Unidade do Trauma é um projeto em desenvolvimento que implica uma série de fatores, que vai desde a subdivisão de serviços especializados até a sua complexidade: fluxos de trabalho, materiais e insumos, estrutura física, visando ao controle de infecção nas unidades e no hospital como um todo, tendo como premissa de análise a RDC n. 50 de 21 de fevereiro de 2002, da Agência Nacional de Vigilância Sanitária.

A Unidade de Trauma Ortopedia está sendo implantada na Santa Casa de Campo Grande com o objetivo de fortalecer a rede de atenção à urgência e emergência no âmbito da macrorregião de 
Campo Grande, considerando que o hospital é o único credenciado pelo Ministério da Saúde como Hospital Tipo III em Urgência e está inserido no Plano de Ação da Rede de Atenção às Urgências do Estado de Mato Grosso do Sul.

Com a abertura dessa nova unidade (exclusiva para o SUS) a instituição passa a proporcionar o aumento da oferta de serviços à rede, pois contará com 100 leitos de internação, 10 leitos de UTI, cinco salas cirúrgicas e 18 leitos de observação. 0 anexo em construção (unidade de trauma ortopédico) está localizado fora do edifício sede. Portanto, essa nova Unidade necessita de equipamentos e mobiliários específicos para o seu funcionamento. Quando essa nova unidade entrar em funcionamento, estima-se que a instituição irá produzir mais: 5.800 internações/ano; 6.000 cirurgias/ano; 500 internações de pacientes críticos/ano; 3.500 atendimentos de Urgência e Emergência/ano, além de outros serviços de diagnóstico.

Na execução da reforma da unidade também está prevista a construção de uma passarela metálica para "ligar" a Santa Casa com a Unidade de Trauma Ortopedia, tendo em vista que a nova unidade irá utilizar os serviços de lavanderia, cozinha, central de esterilização de materiais entre outros, que estão concentrados no edifício sede.

\section{CONSIDERAÇÕES FINAIS}

A investigação realizada evidencia em seus objetivos os atendimentos que serão realizados na Unidade de Trauma ortopédico com vistas ao Desenvolvimento Local, em especial, os aqui elencados. Ambos têm como foco fomentar o protagonismo individual e coletivo a partir de suas vivências, na construção da territorialidade, utilizando recursos e potencialidades locais, articuladas com políticas públicas e parcerias com organizações da sociedade civil organizada. 
Compreende-se que, com a abertura da Unidade de Trauma ortopédico na Santa Casa, o Pronto Socorro da instituição deverá receber os pacientes e os diagnosticados com trauma deverão ser encaminhados para a unidade em estudo. Isso implicará melhoria no atendimento das outras especialidades que derem entrada no hospital, e, com isso, os profissionais deverão realizar o atendimento com mais humanidade e agilidade no processo.

As dificuldades encontradas foram o alinhamento das informações referentes aos serviços que serão realizados na Unidade de Trauma ortopédico, pois estão sendo feitos estudos referentes aos serviços que já estão sendo realizados na instituição, pois, com o aumento da demanda, os serviços deverão passar por algumas adequações/investimentos.

A instituição tem como foco principal o atendimento da população de forma humanizada, sendo um dos fatores relevantes para a implantação da Unidade de Trauma ortopédico, com isso, estudos estão sendo realizados para que os investimentos necessários para sua implantação sejam destinados junto com as instituições públicas.

Relacionando a pesquisa com as demandas previstas, a instituição terá o desafio de, junto com os órgãos públicos e demais instituições, realizar medidas contributivas que deverão ser uma forma da Unidade de Trauma ortopédico, tendo em vista que esta unidade será destinada exclusivamente aos usuários do Sistema Único de Saúde.

Em posse dos dados obtidos, foi possível perceber que, para a abertura da Unidade de Trauma ortopédico, a instituição, junto com os órgãos públicos, deverá realizar investimentos em suas estruturas físicas, materiais e equipamentos, tanto para a unidade em estudo quanto para os serviços que darão apoio a essa unidade.

Há de se reconhecer que a Unidade de Trauma ortopédico será um grande benefício para o Município de Campo Grande e 
para o Estado de Mato Grosso do Sul, pois a rede de atenção à saúde terá mais leitos disponíveis para o atendimento da população. É seguindo essa perspectiva que a instituição deve continuar lutando pela implantação dessa unidade, tendo em vista que esta será de grande necessidade do setor da saúde, por beneficiar significativamente a população nesse processo, ou seja, deve buscar por meio do empoderamento local articular políticas e desenvolver ações que favoreçam o Desenvolvimento Local.

\section{REFERÊNCIAS}

ALBAGLI, Sarita; MACIEL, Maria Lúcia. Informação e conhecimento na inovação e no Desenvolvimento Local. Ciência da Informação, Brasília, DF, v. 33, n. 3, p. 9-16, set./dez. 2004.

ÁVILA, Vicente Fideles. Pressupostos para formação educacional em desenvolvimento local. Interações - Revista Internacional de Desenvolvimento Local, Campo Grande, MS, v. 1, n. 1, p. 63-76, set. 2000. BRASIL. Ministério da Saúde. Secretaria de Assistência à Saúde. Departamento de Redes e Sistemas Assistenciais. Gestão hospitalar: uma visão atual do futuro. Brasília: Ministério da Saúde, 2000.

CHERUBIM, N. A.; SANTOS, N. A. Administração hospitalar: fundamentos. 3. ed. São Paulo: Loyola, 2002.

ELIZALDE, Antonio. Desarrollo a escala humana: conceptos y experiências. Interações - Revista Internacional de Desenvolvimento Local, Campo Grande, MS, v. 1, n. 1, p. 51-62, set. 2000.

FONTAN, Jean-Marc; VIEIRA, P. F. Por um enfoque sistêmico, ecológico e "territorializado". In: TREMBALY, G.; VIEIRA, P. F. (Org.). O papel da universidade no desenvolvimento local. Florianópolis, SC: Secco, 2011.

FOLLAND, Sherman; GOODMAN, Allen C.; STANO, Miron. A economia da saúde. 5. ed. Porto Alegre, RS: Bookman, 2008.

KLIKSBERG, Bernardo. Capital social e cultura: as chaves esquecidas do desenvolvimento. Rio de Janeiro: Programa de Promoção de Reforma Educativa na América Latina e Caribe-PREAL/Brasil, 2002. 
LE BOURLEGAT, C. A. Desenvolvimento local na abordagem territorial do atual sistema-mundo. In: TREMBALY, G.; VIEIRA, P. F. (Org.). O papel da universidade no Desenvolvimento Local. Florianópolis, SC: Secco, 2011. MARTINS, Sérgio Ricardo Oliveira. Desenvolvimento Local: questões conceituais e metodológicas. Interações - Revista Internacional de Desenvolvimento Local, Campo Grande, MS, v. 3, n. 5, p. 51-59, set. 2002. PORTELA, M. C. et al. Caracterização assistencial de hospitais filantrópicos no Brasil. Revista de Saúde Pública, Rio de Janeiro, v. 38, n. 06, dez. 2004.

RAFFESTIN, Claude. 0 que é o território? In: Por uma geografia do poder. São Paulo: Ática, 1993. p. 144-220.

SANTA CASA. Relatório de atividades. Campo Grande, MS, 2014.

SANTA CASA. Histórico da Associação Beneficente de Campo Grande, Santa Casa. Documento avulso. Campo Grande, MS, [s./d.].

SANTOS, Milton. O retorno do território. In: SANTOS, M. et al. Território: globalização e fragmentação. São Paulo: Hucitec, 1994.

SANTOS, Milton. O espaço do cidadão. São Paulo: HUCITEC, 2002.

SOUZA, Maria Adélia Aparecida de. O lugar de todo mundo: a geografia da solidariedade. Conferência apresentada no I ENCONTRO INTERNACIONAL DE GEOGRAFIA DA BAHIA, Salvador, BA, 1997.

TUAN, Yi-Fu. Topofilia - um estudo da percepção, atitudes e valores do meio ambiente. São Paulo: Difel, 1980. 